\title{
Prevalencia del síndrome metabólico en estudiantes de medicina de la Universidad Autónoma de Aguascalientes
}

Prieto-Macías Jorge*, Franco-Díaz de León Raúl*, Terrones-Saldívar Ma del Carmen*, Rosas-Cabral Alejandro*, Reyes-Robles Martha Elena*, Aranda-González María Fernanda**, Escobedo-Duarte Diana Lizbeth**, Gómez-Moreno Carolina**, González-González César**, Medina-Campos Claudia Rosalía**,

\section{Resumen}

- El síndrome metabólico (SM), es un problema de salud pú- blica que afecta cada vez más a la población de jóvenes. La

- Encuesta Nacional de Salud y Nutrición 2006 en población

- mexicana documentó sobrepeso y obesidad en 70\%, diabetes

- mellitus en 7\% e hipertensión arterial en 30.8\%.' En estudian-

- tes universitarios se ha encontrado una prevalencia del 3.7\%.2

- El objetivo de este estudio fue documentar la prevalencia del

- SM en estudiantes de medicina de la Universidad Autónoma

- de Aguascalientes (UAA). Metodología: Estudio descriptivo,

- no experimental, transversal. Se efectuó en un grupo de es-

- tudiantes con edades entre los 17 a 26 años, de agosto a

- diciembre de 201 I. Mediante un muestro de conveniencia, se

- estudiaron a 230 estudiantes, a los que se determinaron los

- criterio de SM de acuerdo con ATP III ${ }^{3}$. Resultados: de los 230

- estudiantes, 108 fueron mujeres y 128 hombres. La preva-

- lencia del SM fue de 15.2\% (35 estudiantes); 22 estudiantes

- (9.56\%) tuvieron 3 criterios y otros 13 alumnos, 4 criterios.

- Los factores más frecuentes fueron: índice de masa corpo-

- ral $\geq 25$ (IMC) en el 80\%, lipoproteínas de alta densidad

- disminuídas (HDL) en el 60\%, hipertensión arterial sistémica,

- $51 \%$, hipertrigliceridemia $45 \%$ y obesidad abdominal 45

- \%. Conclusión: El SM y los criterios de diagnóstico del mismo,

- pueden encontrase también en edades tempranas de la vida.

- El criterio más frecuente documentado fue el sobrepeso. Es-

- tos resultados nos deben motivar a efectuar programas de

- prevención en nuestra universidad. LUX MÉDICA, AÑO 7, NÚMERO 22,

- SEPTIEMBRE-DICIEMBRE 2012 PP 3-9

\section{Abstract}

Metabolic syndrome (MS) is a public health problem affecting more and more young people. The national survey of health and nutrition 2006 in mexican population documented overweight and obesity in 70\%, diabetes mellitus in 7\%, and hypertension in 30.8\%.' in academic students has found a prevalence of $3.7 \% .^{2}$ the objective of this study was to document the prevalence of MS in students of Medicine at the University of Aguascalientes (UAA). Methodology: Descriptive, not experimental, cross- sectional study. It was a group of students aged between 17 to 26, from August to December 201 I. Sampling of convenience, 230 students were studied, that the criterion of MS according to ATP $I I I \mathrm{~m}^{3}$ were determined. Results: 230 students, 108 gender female and 128 male. The prevalence of MS was 15.2\% (35 students); 22 students (9.56\%) had 3 criteria and other 13 students, 4 criteria. The most common factors were: Body mass index BMI $\geq 25$ (BMI) in $80 \%$, decreased high-density lipoprotein (HDL) in $60 \%$, systemic hypertension in $51 \%$, hypertriglyceridemia $45 \%$ and 45\% abdominal obesity. Conclusion: The MS and the same diagnostic criteria, can be also found at early ages of life. The most common criterion was overweight. These results should motivate to carry out prevention programs at the University. LUX MÉDICA, ANNO 7, NÚMERO 22, SEPTIEMBRE-DICIEMBRE 2012 PP 3-9

Palabras clave: síndrome metabólico, estudiantes, medicina.

Key words metabolic síndrome, students, medicine

$$
\text { • }
$$
$\cdot$ $\div$ 


\section{Introducción}

El síndrome metabólico (SM) es un concepto clínico que se caracteriza por la asociación de intolerancia a la glucosa, hipertensión arterial primaria, obesidad central, dislipidemia, hiperlipidemia, hiperfibrinogenemia, microalbuminuria y ateroesclerosis. La resistencia a la insulina parece ser el factor común del síndrome metabólico de acuerdo al National Cholesterol Education Program in the Adult Treatment Panel III (NCEP-ATPIII) ${ }^{1-4}$

Este síndrome, de manera silenciosa, se está convirtiendo en un problema creciente de salud pública en nuestro país, incluso en población joven, asociado a un incremento de la prevalencia de diabetes tipo 2 y de enfermedades cardiovasculares ${ }^{5-8}$. Existen escasos reportes sobre la prevalencia de SM en estudiantes de medicina. En el año de 2007 González y colaboradores, reportaron una prevalencia de $3.7 \%$ de SM en un grupo de estudiantes universitarios de la Universidad de Veracruz ${ }^{2}$. El interés de este trabajo fue identificar la prevalencia del SM en los estudiantes de la carrera de Medicina del Centro de Ciencias de la Salud de la Universidad Autónoma de Aguascalientes (UAA).

\section{| | | | | | | | | | | | | | | | | | | | | | | | | | | | | | | | | | | | | | | | | | | | | | | | | | | | | | | | | | | | | | | | | | | | | | | | | | | | | | | | | | | | | | | | | | | | | | | | | | | | | ||}

\section{Material y métodos}

Estudio observacional, descriptivo y transversal. De un total de 670 estudiantes inscritos en el semestre agosto-diciembre 2011 en la carrera de medicina, se incluyeron, mediante muestreo por conveniencia, a 230 alumnos de diferentes semestres de la carrera de medicina del Centro de Ciencias de la Salud de la Universidad Autónoma de Aguascalientes. A los 230 estudiantes se les realizó, previo consentimiento informado, determinación de glucosa sérica, lípidos séricos, HDL, triglicéridos, colesterol sérico total. Las muestras fueron obtenidas por personal capacitado y fueron procesadas en un laboratorio certificado. Se registró además, la presión arterial, en dos ocasiones, con un intervalo de quince minutos, ambas en posición sedente, con un periodo de descanso previo a la toma de cinco minutos, con un esfigmomanómetro aneroide marca Adex, con mango universal para adultos. La toma fue realiza- da por una sola persona con entrenamiento para realizarla. La cifra final resultó del promedio de las dos mediciones con intervalos de 10 minutos entre ellas. Se midió el perímetro abdominal, y se registró el peso en una báscula y talla para obtener el índice de masa corporal. Además se consideraron otras variables como edad, género, consumo o no de tabaco, consumo o no de alcohol y la presencia o ausencia de sedentarismo, los antecedentes familiares de hipertensión arterial, sobrepeso, obesidad y diabetes mellitus. No se determinó fibrinógeno sérico, ni la presencia de microalbuminuria a pesar de ser criterios incluidos en la última versión del NCEP-ATPIII. Con los datos obtenidos, se integró el diagnóstico de síndrome metabólico cuando algún estudiante reunía tres o más criterios de acuerdo al NCEP - ATPIII ${ }^{3}$ (ver tabla 1). Los resultados se presentan con medidas de tendencia central y se utilizó la $x^{2}$ cuando fue necesario. 


\section{Tabla I}

Criterios del NCEP-ATPIII para integrar el diagnóstico de síndrome metabólico ${ }^{6}$

\begin{tabular}{|c|c|}
\hline \multicolumn{2}{|c|}{$\begin{array}{l}\text { National Cholesterol Education Program in the Adult Treatment Panel III (NCEP - ATPIII) } \\
\text { El síndrome metabólico es identificado con la presencia de tres o más factores de riesgo }\end{array}$} \\
\hline FACTOR DE RIESGO & NIVEL PREDICTOR \\
\hline Obesidad Abdominal & Perímetro abdominal \\
\hline Hombre & $>102 \mathrm{~cm}$ \\
\hline Mujer & $>88 \mathrm{~cm}$ \\
\hline Triglicéridos & $\geq 150 \mathrm{mg} / \mathrm{dL}$ \\
\hline \multicolumn{2}{|l|}{ Colesterol HDL } \\
\hline Hombre & $<40 \mathrm{mg} / \mathrm{dL}$ \\
\hline Mujer & $<50 \mathrm{Mg} / \mathrm{dL}$ \\
\hline Colesterol LDL & $>100 \mathrm{mg} / \mathrm{dL}$ \\
\hline Presión arterial & $\geq 130 / 85 \mathrm{mmHg}$ \\
\hline Glucosa en ayuno & $\geq 110 \mathrm{mg} / \mathrm{dL}$ \\
\hline Estado proinflamatorio & No es posible su identificación en la evaluación clínica de rutina \\
\hline Estado protrombótico & \\
\hline
\end{tabular}

\section{Resultados}

Se incluyeron a 230 estudiantes de diferentes semestres de la carrera de medicina, con un promedio de edad de 20.4 años, con un rango de 17 a 26 años. La distribución por género, fue de 102 mujeres (44.3\%) y 128 hombres $(55.7 \%)$. Se encontró una prevalencia de uso de tabaco del 13\% (30/230), y del uso de alcohol de 47.8\% (110/230). En la mayoría de los alumnos, se les identificó al menos un criterio del SM. Solo el $30.6 \%$ de los estudiantes no tuvieron ningún criterio de riesgo para SM. Se encontraron disminuidos los niveles de HDL en el $22 \%$ y los triglicéridos elevados en el $6.9 \%$ de los estudiantes. Estas alteraciones se presentaron con un predominio del sexo masculino 2:1 en comparación a la mujer.

La prevalencia de síndrome metabólico en este estudio fue de $15.2 \%$ (35/230), es decir que al menos cumplieron con tres de los criterios definidos por el NCEP-ATPII; en la mayoría de los estudiantes con SM, se identificaron cuatro criterios del NCEPATP-III. De los 35 estudiantes identificados con SM, 23/35 (65.7\%) fueron del sexo masculino y $12 / 35$ del sexo femenino (34.3\%).

Los criterios del ATP-III más frecuentes fueron: el índice de masa corporal mayor de 25, disminución en la concentración sérica de HDL y el perímetro abdominal e hipertensión, en 28/35, 21/35, 16/35 y $12 / 35$ respectivamente. Tabla 2 .

\section{Tabla 2}

Distribución de la frecuencia de los criterios encontrados en 35 estudiantes con SM de la carrera de medicina de la UAA según género

\begin{tabular}{|c|c|c|c|}
\hline Criterios diagnósticos positivos & Número de estudiantes & Mujeres & Hombres \\
\hline$I M C \geq 25$ & 28 & 10 & 18 \\
\hline $\mathrm{HDL}$ & 21 & 8 & 13 \\
\hline Perímetro abdominal & 16 & 5 & 11 \\
\hline HTAS Sistólica & 12 & 5 & 7 \\
\hline HTAS Diastólica & 16 & 5 & 11 \\
\hline Triglicéridos $>150 \mathrm{mg} / \mathrm{dl}$ & 16 & 6 & 10 \\
\hline Glicemia $>110 \mathrm{mg} / \mathrm{dl}$ & 4 & 4 & 0 \\
\hline
\end{tabular}


El consumo de tabaco se encontró positivo en 5 estudiantes con SM (14.2\%), el del alcohol en el $57.1 \%(20 / 35)$ y el sedentarismo en $42.8 \%$ (15/35). Figura 1

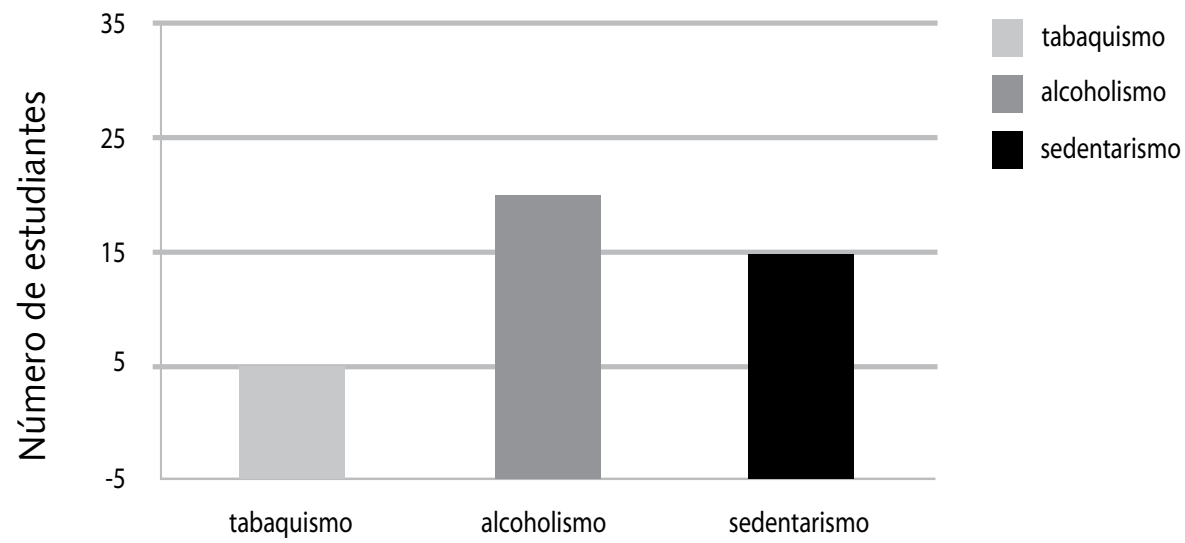

Figura 1. Distribución de factores relacionados con estilo de vida, en 35 estudiantes con síndrome metabólico

Tuvieron sobrepeso/obesidad, de acuerdo con el índice de masa corporal (IMC), 72 de los 230 estudiantes evaluados, que corresponde al $31.3 \%$. En estos estudiantes, en 28 de ellos (38.8\%), se integró el diagnóstico de SM. A su vez, el sobrepeso/obesidad estuvo presente como criterio diagnóstico en 28/35 estudiantes con SM, lo que representa el $80 \%$ Tabla 3.

\section{Tabla 3}

Distribución del ÍMC en 230 Estudiantes de Medicina de la UAA según género

\begin{tabular}{|lccccc|}
\hline & Peso menor del normal & Peso Normal & Sobrepeso & Obesidad & Total \\
\hline Número de estudiantes & 14 & 144 & 56 & 16 & 230 \\
\hline Mujeres & 9 & 73 & 17 & 3 & 102 \\
\hline Hombres & 5 & 71 & 39 & 13 & 128 \\
\hline Porcentaje & 6.08 & 62.60 & 24.34 & 6.95 & 100 \\
\hline
\end{tabular}

Otro de los componentes del SM encontrado en nuestros alumnos fue la hipertensión, la cual fue considerada presente según la definición del NCEP-ATP III, cuando la presión arterial fue superior a 130/85 mm Hg. Se documentó hipertensión en 47 de los 230 estudiantes evaluados, que corresponde al $20.4 \%$, con pre- dominio del sexo masculino con relación 2:1 en comparación con la mujer. De estos estudiantes, en 18 de ellos (38\%), se integró el diagnóstico de síndrome metabólico. A su vez, la hipertensión estuvo presente como criterio diagnóstico en 18/35 estudiantes con SM, lo que representa el 51.4\% .Tabla 4. 


\section{Tabla 4}

Distribución de la presión arterial en 35 estudiantes con síndrome metabólico de la carrera de medicina de la UAA

\begin{tabular}{|l|c|c|c|c|c|c|c|c|}
\hline & Sólo Sistólica & $\%$ & Sólo Diastólica & $\%$ & Ambas & \% & Total & $\%$ \\
\hline Mujeres & 2 & 11.1 & 1 & 5.5 & 4 & 22.2 & 7 & 38.8 \\
\hline Hombres & 0 & 0.0 & 5 & 27.7 & 6 & 33.3 & 11 & 61.2 \\
\hline Total & 2 & 11.1 & 6 & 33.3 & 10 & 55.5 & 18 & 100 \\
\hline
\end{tabular}

La hiperglicemia se documentó solamente en cuatro mujeres del total de 230 $(1.7 \%)$, sin embargo, las cuatro tuvieron síndrome metabólico.
Los antecedentes heredofamiliares más frecuentes entre los alumnos con síndrome metabólico fueron los de diabetes mellitus e hipertensión, ambos antecedentes estuvieron presentes en el $77 \%$ de los casos.

\section{Tabla 5}

Distribución de los antecedentes heredofamiliares en $\mathbf{3 5}$ estudiantes con síndrome metabólico.

\begin{tabular}{|lcccccc|}
\hline & DM & HTAS & Nefropatía & Retinopatía & Otros \\
\hline Mujeres & 8 & 9 & 1 & 3 & 3 \\
\hline Hombres & 19 & 18 & 2 & 5 & 12 \\
\hline Total & 27 & 27 & 3 & 8 & 15 \\
\hline
\end{tabular}

\section{Discusión}

La Encuesta Nacional de Salud y Nutrición 2006 ha reportado una prevalencia de SM en nuestro país, en adultos mayores de 20 años de $36.8 \%$, si se toma en cuenta los criterios del NCEP; también refiere una prevalencia de síndrome metabólico de $23.8 \%$ (IC 95\% 21.2-26.5\%) en el grupo de edad entre 20 y 39 años de edad, grupo etario al que pertenecen nuestros estudiantes. ${ }^{9}$

Si bien existen diversos trabajos en la literatura sobre la prevalencia del SM en estudiantes universitarios, existen pocos trabajos que estudien de manera específica la prevalencia de este síndrome en estudiantes de medicina. Estos trabajos han reportado prevalencias significativamente menores a las encontradas por nosotros en estudiantes de medicina, las cuales se encuentran entre el $1 \%$ en estudiantes universitarios chilenos ${ }^{10}$, en Polonia reportaron un $2.65 \%$ de prevalencia de $S M^{11}$, en Colombia Feliciano y colaboradores estudiaron 249 estudiantes universitarios con edades entre los 15 y 20 años y encuentraron una prevalencia de $2.4 \%{ }^{12}$. Otros autores como Huang y colaboradores encontraron en estudiantes de la Universidad de Kansas, una prevalencia en este rango ${ }^{13}$ y González Deschamps en la Universidad Veracruzana estudió 402 estudiantes de diferentes carreras y reportó una prevalencia del $3.7 \%{ }^{2}$. Estos datos indican una prevalencia significativamente menor que la encontrada por nosotros del $15.2 \%$, lo cual nos obliga a realizar estudios prospectivos que nos permitan definir los factores de riesgo que están determinando esta elevada prevalencia, para posteriormente diseñar las estrategias adecuadas que permitan, en primera instancia disminuir la prevalencia del SM en nuestros estudiantes y posteriormente que 
ello influyan en la disminución de la prevalencia de esta enfermedad en la población general de nuestro estado.

Existe un solo trabajo reportado por $\mathrm{Ni}$ llakupt y Viravathana que estudió la prevalencia de SM en estudiantes de medicina, ellos incluyeron a 95 cadetes de la escuela militar de Medicina de Tailandia y encuentraron una prevalencia del $1.04 \%$ de $\mathrm{SM}^{14}$; esto sugiere que ambos estudios no son comparables, debido a que en primer lugar la población del estudio de Nulakupt se realizó en estudiantes de raza asiática, la cual tiene un fondo genético notablemente diferente al de la población mexicana mestiza, estudiada por nosotros y constituida al menos por tres componentes, un amerindio, un europeo y uno africano, aunque en nuestra región son predominantes el componente amerindio y europeo; así mismo la condición militar de los estudiantes Tailandeses, los obliga a un acondicionamiento físico más intenso y de manera regular, lo cual no es comparable con nuestros alumnos en los cuales el porcentaje de sedentarismo resulto elevado, aunado a que el porcentaje de sobrepeso y obesidad encontrados en alumnos de medicina tailandeses fue del $21.88 \%$ y $15.62 \%$ cifras notoriamente diferentes de $80 \%$ encontrado en nuestros alumnos ${ }^{15}$.

No obstante estas diferencias, sus hallazgos resultan relevantes, debido a que ellos encontraron que el componente del SM más frecuente, fue el descenso en la cifra de HDL, seguido de la prevalencia de hipertensión, similar a lo encontrado por nosotros, en que el descenso del colesterol HDL fue el segundo componente del SM más frecuente, este dato es concordante con lo reportado en otros estudios como la ENSAUT 2006 en la que reportaron un 76.3\% de prevalencia de descenso de HDL, pero notablemente mayor que lo encontrado por González-Deschamps, Huang y Feliciano con $28.9 \%$, $24 \%$ y $13.3 \%$ respectivamente ${ }^{12-14}$.
Casi el 95\% de los individuos diagnosticados con síndrome metabólico por nosotros, tienen por lo menos un antecedente heredofamiliar relevante para enfermedad cardiovascular o metabólica. El antecedente que más predominó fue la hipertensión arterial sistémica, seguida del antecedente de diabetes mellitus tipo 2 .

El antecedente heredofamiliar para diabetes mellitus fue identificado en 162 de los 230 estudiantes, y en 27 de los 35 alumnos con síndrome metabólico, lo que representó 1.44 veces más posibilidades de tener síndrome metabólico. Por otro lado, el antecedente de hipertensión arterial sistémica fue positivo en 149 de los 230 estudiantes evaluados, y en 27 de los 35 estudiantes con síndrome metabólico, lo que representó 1.8 veces más posibilidades de tener síndrome metabólico.

Es de llamar la atención que el sobrepeso/obesidad fue identificado en el $80 \%$ de los alumnos con síndrome metabólico, que representó, en este estudio, 8.7 veces más posibilidades de que un estudiante con sobrepeso/obesidad, tuviera el síndrome metabólico. El dato de hipertensión arterial sistémica se registró en un poco más de la mitad de los estudiantes con síndrome metabólico, y representó 3.7 veces más el riesgo del síndrome metabólico.

Tanto el consumo de tabaco, observado en el $14.2 \%$, como la ingesta de alcohol $(57 \%)$ y el sedentarismo (42.8\%), fueron hábitos en el grupo estudiado, con predominio en el sexo masculino.

No debemos olvidar al grupo de estudiantes que tuvieron uno o dos criterios de la ATP-III, que aún cuando no tienen el diagnóstico de síndrome metabólico, de no modificar el estilo de vida, sobre todo, la disminución de peso, son candidatos potenciales de complicaciones en un futuro no lejano. 


\section{Conclusiones}

Aunque la mayoría de los estudiantes universitarios pueden ser considerados "sanos", algunos de ellos pueden ser portadores de factores de riesgo para el desarrollo de síndrome metabólico y por ende enfermedad cardiovascular, sin ser sintomáticos en el momento actual; no obstante muchos de estos factores de riesgo son modificables por lo que es necesario determinar su prevalencia para que se implanten medidas preventivas en esta población.

El SM y los criterios de diagnóstico del mismo aparecen con elevada frecuencia en individuos jóvenes. El criterio más frecuente en nuestra población fue el sobrepeso. Estos resultados nos deben motivar para efectuar programas de prevención en nuestra UAA.

\section{Bibliografía}

1. Encuesta Nacional de Salud y Nutrición, México D.F. 2006. http://www.insp.mx/encuesta-nacional-salud-y-nutricion-2006.html

2. González Deschamps E., Palmeros Exsome Carolina, Villanueva Sánchez Javier, Torres Flores Beatriz, Bastida Sara, Vaquero Rodrigo María Pilar, SánchezMuniz Francisco José. Prevalencia de síndrome metabólico y su asociación con el indice de masa corporal en estudiantes universitarios, Revista Medicina clínica, 2007,129(20):766-769

3. Third Report of the National Cholesterol Education Program (NCEP) Expert Panel on Detection, Evaluation, and Treatment of High Blood Cholesterol in Adults (Adult Treatment Panel III) Final Report, Journal of the American of the Heart Association, 15244539, 2002.

4. González Chávez A., Lavalle González F. J., Ríos González J. J., Síndrome Metabólico y Enfermedad Cardiovascular. Criterios clínicos aplicables a la práctica médica., Intersistemas Editores, México 2004, pp 1-10.

5. Encuesta Nacional de Salud y Nutrición, Resultados por Entidad Federativa, Aguascalientes, 2006.

6. World Health Organization. Definition, diagnosis and classification of diabetes mellitus and its complications. Report of a WHO consultation. Geneve: WHO; 1999.

7. Zimmet P., MM Alberti K. G., Serrano Ríos M., Una nueva definición mundial del síndrome metabólico propuesta por la Federación Internacional de Diabetes: fundamento y resultados., Rev Esp Cardiol. 2005;58:1371-1376.
8. Motillo S., Filion K., Genest J., Joseph L., Pilote L., Poirier P., Rinfret S., Schiffrin E., Eisenberg M., The metabolic syndrome and cardiovascular risk: a systematic review and meta-analysis, Journal of the American College of Cardiology (2010),56(14): 11131132

9. Hernández Tamayo, M. \& cols. Comportamiento de variables clínicas, antropométricas y de laboratorio en síndrome metabólico. Revista Electrónica de las Ciencias Médicas, ISSN:1727-897X. España, 2011.

10. Palomo IF, Torres Gl, Alarcón MA, Moragaño PJ, Leiva $E$, Mujica V. Alta prevalencia de factores de riesgo cardiovascular clásicos en una población de estudiantes universitarios de la región centro-sur de Chile. Rev Esp Cardiol 2006;59:1099-1105

11. Koziarska-Rosciszewska M, Panasiuk M, Cypryk K, Prevalence of metabolic syndrome and its components in the Young adult-students of universities in Lodz, Poland. 2010;16(4):277-283.

12. Feliciano-Alfonso JF, Olimpo-Mendivil C, Sierra-Ariza ID, Pérez CE. Cardiovascular risk factors and metabolic syndrome in a population of young students from the National University of Colombia. Rev Assoc Med Bras 2010;56(3):293-298

13. Huang TT, Shimel A, Lee RE, Delancey $W$, strother $\mathrm{ML}$, Metabolic risk among college students: prevalence and gender differences. Metab Syndr Related Disord. 2007;5(4):365-372.

14. Nillakupt K; Viravathana N. A survey of metabolic síndrome and its components in Thai medical cadets. J Med Assoc Thai. 2010;92S6:179-185 


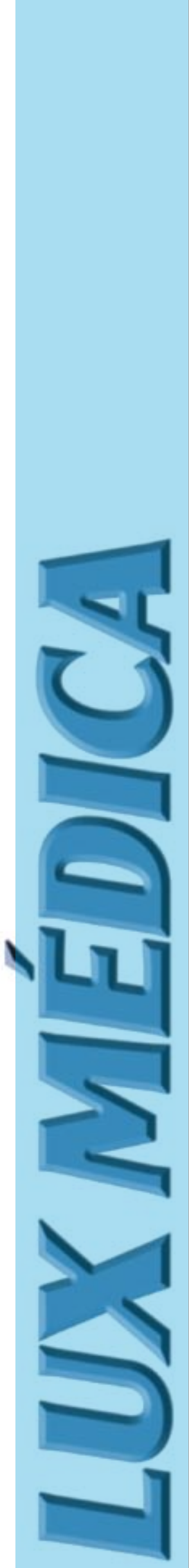

